\title{
Patient journey pain points in spondyloarthritis and psoriasis: The case of Greece
}

\author{
Enkeleint Aggelos Mechili ${ }^{1,2}$, Katerina Nikitara ${ }^{1}$, Charis Girvalaki ${ }^{1}$, Christina Kyriakos ${ }^{1}$, Constantine Vardavas ${ }^{1}$
}

\section{AFFILIATION}

1 Medical School, University of Crete, Greece

2 Department of Healthcare, Faculty of Public Health, University of Vlora, Vlora, Albania

\section{CORRESPONDENCE TO}

Enkeleint Aggelos Mechili. Medical School, University of Crete, Heraklion

715 00, Greece. E-mail: mechili@uoc.gr

\section{KEYWORDS}

psoriasis, screening, healthcare system, treatments

Received: 23 July 2019, Revised: 20 September 2019, Accepted: 25 October 2019

Popul. Med. 2019;1(November):4

https://doi.org/10.18332/popmed/113401

\begin{abstract}
For the diseases spondyloarthritis ( $\mathrm{SpA}$ ) and psoriasis, the patient's journey commences when the first symptoms present themselves. However, many patients ignore the initial symptoms, until the moment they become unbearable. Additionally, due to GPs/family physicians and primary healthcare providers' lack of knowledge, both SpA and psoriasis remain underdiagnosed, and patients need to undertake multiple visits and health examinations to get correctly diagnosed. With regard to appropriate treatment, a combination of pharmacotherapy, counselling and education
\end{abstract}

is useful to improve the patient's quality of life, with healthcare professionals ensuring the patient's adherence to the therapy. In Greece, the lack of health protocols and health technology assessment procedures create a burden in the screening, diagnosis and treatment of patients with SpA and psoriasis. Based on the aforementioned, the existence of a comprehensive, patient-centred and integrated healthcare system is of crucial importance so that patients with chronic conditions can have quality healthcare, better prognosis and reduced cost of care.

\section{COMMENTARY}

Despite the fact that skin disorders lead to chronicity, usually, people consider them less important than other diseases, as they are not life-threating. Evidence of the peculiarities of seronegative spondyloarthropathies (SpA) and psoriasis (including psoriatic arthritis) has increased worldwide, as indicated by the large number of recent publications on this topic ${ }^{1}$.

SpA is a heterogeneous group of chronic inflammatory rheumatic diseases present as inflammatory back pain, asymmetric peripheral arthritis, enthesitis, dactylitis, and tenosynovitis. SpA can even cause structural complications to spine and sacroiliac joints. Genetic issues, microbial triggers, inflammation of bone marrow and enthesial structures are critical determinants of the disease $\mathrm{e}^{2,3}$. Prevalence of SpA varies among populations and geographical areas. Worldwide prevalence is estimated to be from $0.5 \%$ to $1.9 \%{ }^{4}$. According to the ESORDIG study, Greece has a lower prevalence of SpA compared to other countries $(0.49 \%)^{5}$. However, up-to-date data are lacking, yet needed in public policy planning.

Psoriasis is an autoimmune chronic inflammatory disorder of the skin. Plaque psoriasis is the most common manifestation of the disease ( $85 \%$ frequency). Frequency of occurrence is from 2 to $4 \%$ worldwide, with around 125 million people living with psoriasis6. Psoriatic arthritis (also known as inflammatory arthritis) affects $4 \%$ to $30 \%$ of individuals with psoriasis ${ }^{7}$. Prevalence of non-mandatory registration ranges from $0.09 \%$ to $11.4 \%$. The disease onset can be at any age but with two peaks in the age groups 1622 and 57-60 years ${ }^{8}$. In general, new cases of psoriasis and psoriatic arthritis are higher in Europe and USA and lower in Latin America ${ }^{9}$. In Greece, epidemiological evidence of the parameters of this disease and its most frequent symptoms and target points have been examined ${ }^{10}$. In 2013, a retrospective study demonstrated that among 278 patients with psoriasis, $86 \%$ were diagnosed with plaque psoriasis, $5 \%$ guttate, $2 \%$ palms and soles, $2 \%$ inverse, $1 \%$ pustular and $4 \%$ with psoriasis of more than one type ${ }^{11}$.

Both SpA and psoriasis are severe health problems and are related to a lower quality of life. Additionally, they are closely connected with low physical and emotional status (stress, depression, anxiety, and sometimes even suicidal 
ideation ${ }^{8,12}$. Except for the aforementioned problems, the diseases are linked to impaired productivity ${ }^{8,13,14}$.

Based on all the above, the aim of this brief policy article is to depict SpA and psoriasis patients' journey within a healthcare system and the steps needed to improve this journey.

\section{Patient journey}

For both diseases, the patient's journey commences when the first symptoms are presented. The first contact point for patients with SpA is the general practitioner while for the psoriasis patients a general practitioner or dermatologist (Contact Point 1). However, in both cases first contact with the healthcare system takes place when the signs and symptoms deteriorate, with discomfort and pain being unbearable leading to difficulties in performing daily tasks. This delay in the first contact is a result of many factors such as misconception of the seriousness of the disease and familial experience of the disease etc. All previously mentioned points could be defined as pre-contact pain points (Pain Point 1).

The core aim of health policy-makers and healthcare system must be the modification of these barriers with patient engagement and population awareness being at the centre of this reform. Within this context the Greek public healthcare (PHC) system has been under reform in the last years ${ }^{15}$, by mainly shifting the role of the GPs/family physicians as the system gatekeeper. Despite the political will for this reform, GPs/family physicians may not have sufficient training and education as well as the appropriate tools for disease diagnosis ${ }^{5}$. Due to the lack of knowledge and competencies, GPs/family physicians may ignore the severity of the symptoms and misdiagnose the disease or refer the patient to the appropriate specialist.

Unfortunately, in Greece patients may directly visit a rheumatologist without initially visiting a GP or family doctor (or another primary health care practitioner). Most of the times, the specialist is the first contact point for these patients. A direct visit to a specialist leads to an important gap in the time frame in which SpA patients seek advice. Due to the visit delay, in most of the cases, the disease has already progressed. Psoriasis symptoms are usually ameliorated with some lifestyle changes such as less stress, healthy diet, smoking cessation, and reduced alcohol consumption ${ }^{16}$. Subsequently, all the delays mentioned above for both SpA and psoriasis are significant for a disease progress and could be characterized as Pain Point 2.

It is well-known that many patients do not pay attention to the numerous initial symptoms, until they become unbearable. In most cases, other healthcare specialists (in gastroenterology, dermatology, orthopaedics, and ophthalmology) are not familiar with screening modalities, and this can lead to perception inconsistencies and problematic diagnosis and management8. Lack of adequate training ${ }^{17}$ for psoriasis leads to lack of awareness resulting in under-diagnosis, inappropriate therapy and unfulfillment of patient needs (Pain Point 2a). Except for misdiagnosing the disease, delayed diagnosis (Pain Point $2 \mathrm{~b}$ ) is another important aspect. Results from the ESORDING study show that SpA patients who visit a rheumatologist have a $91.3 \%$ chance of correct diagnosis compared to only $11.6 \%$ for patients that visit other specialists $(p<0.005)$. This demonstrates the importance of a correct diagnosis by the physician ${ }^{18}$.

When the disease is finally diagnosed well and appropriate treatment is provided by healthcare personnel, the patient's quality of life improves ${ }^{19,20}$. The combination of pharmacotherapy, counselling and education is an effective measure to improve a patient's quality of life. It is of paramount significance that PHC providers operating in primary healthcare be aware of psoriasis and SpA, their management and co-morbidities. After receiving the appropriate treatment and the disease starts to regress, patient satisfaction and physician-patient interaction are improved and the total outcome for both sides is positive (Pain Point a). At this point for both diseases, it is of high importance to provide the most efficient and appropriate treatment. Health protocols have a crucial role (and in Greece authorities are working in this direction) together with the implementation of health technology assessment procedures $^{21-23}$ (Pain Point 3).

Clinicians need to ensure that patient adherence to the proposed therapeutical scheme is followed accordingly (Pain Point 4). Moreover, there should be a transition of the course of treatment (Pain Point 4a), through either a change in the treatment setting or a change in the actual healthcare provider to ensure the continuity of care and the (re-) alignment with appropriate treatment to ensure adherence to pharmacotherapy (Pain Point 4b). In order to increase patient adherence, improved communication with patient and family, simplifying instructions, imparting knowledge, modifying patient beliefs, tailoring education according patient sociodemographic status, are essential ${ }^{24}$. In general, treatment regimens should be offered based on the patient's health status, co-morbidities, impact on quality of life, and response to previous therapies etc.

\section{CONCLUSION}

In order to facilitate the patient's journey, collaboration and synergies between different actors (government, healthcare personnel, patient organizations) are necessary. They should work to provide comprehensive, patientcentred and integrated healthcare services for patients with chronic conditions. This will improve quality of care and access to care, as well as patient outcomes and patient experiences with healthcare systems, while at the same time, the cost of care will be reduced. It is essential that health policymakers in collaboration with healthcare personnel develop guidelines and protocols for disease diagnosis and management. Additionally, healthcare providers' training 
would increase efficiency and improve patient experiences and health status. Finally, patient organizations should continue advocating their rights and provide support to people suffering from psoriasis and SpA.

\section{REFERENCES}

1. Egeberg A, Andersen YMF, Thyssen JP. Prevalence and characteristics of psoriasis in Denmark: findings from the Danish skin cohort. BMJ Open. 2019;9(3):e028116. doi:10.1136/bmjopen-2018-028116

2. Duba AS, Mathew SD. The Seronegative Spondyloarthropathies. Prim Care Clin Off Pract. 2018;45(2):271-287. doi:10.1016/j. pop.2018.02.005

3. Ronneberger M, Schett G. Pathophysiology of Spondyloarthritis. Curr Rheumatol Rep. 2011;13(5):416-420. doi:10.1007/s11926-011-0202-x

4. Bakland G, Alsing R, Singh K, Nossent JC. Assessment of SpondyloArthritis International Society criteria for axial spondyloarthritis in chronic back pain patients with a high prevalence of HLA-B27. Arthritis Care Res (Hoboken). 2013;65(3):448-453. doi:10.1002/acr.21804

5. Trontzas P, Andrianakos A, Miyakis S, et al. Seronegative spondyloarthropathies in Greece: a population-based study of prevalence, clinical pattern, and management. The ESORDIG study. Clin Rheumatol. 2005;24(6):583-539. doi:10.1007/s10067-005-1106-9

6. Albaghdadi A. Current and Under Development Treatment Modalities of Psoriasis: A Review. Endocrine, Metab Immune Disord - Drug Targets. 2017;17(3):189-199. doi:10.2174/1871530317666170804153751

7. Sen R, Hurley JA. Seronegative Spondyloarthropathy. Treasure Island, FL: StatPearls Publishing; 2019. doi:10.1093/med/9780199568741.003.0268

8. World Health Organization. Global report on Psoriasis. Geneva, Switzerland: World Health Organization; 2016. https://apps.who.int/iris/handle/10665/204417. Accessed July 23, 2019.

9. Saad CGS, Gonçalves CR, Sampaio-Barros PD. Seronegative Arthritis in Latin America: A Current Review. Curr Rheumatol Rep. 2014;16(9):438. doi:10.1007/s11926-014-0438-3

10. Rigopoulos D, Gregoriou S, Katrinaki A, et al. Characteristics of psoriasis in Greece: an epidemiological study of a population in a sunny Mediterranean climate. Eur J Dermatol. 2010;20(2):189-195. doi:10.1684/ejd.2010.0869

11. Papadavid E, Katsimbri P, Kapniari I, et al. Prevalence of psoriatic arthritis and its correlates among patients with psoriasis in Greece: results from a large retrospective study. J Eur Acad Dermatology Venereol. 2016;30(10):1749-1752.

CONFLICTS OF INTEREST

The authors have completed and submitted the ICMJE Form for Disclosure of Potential Conflicts of Interest and none was reported.

FUNDING

There was no source of funding for this research. doi:10.1111/jdv.13700

12. Sihgh JA, Strand V. Spondyloarthritis Is Associated with Poor Function and Physical Health-Related Quality of Life. J Rheumatol. 2009;36(5):1012-1020. doi:10.3899/jrheum.081015

13. Haroon NN, Paterson JM, Li P, Inman RD, Haroon N. Patients With Ankylosing Spondylitis Have Increased Cardiovascular and Cerebrovascular Mortality. Ann Intern Med. 2015;163(6):409. doi:10.7326/m14-2470

14. Rudwaleit M, Haibel H, Baraliakos X, et al. The early disease stage in axial spondylarthritis: Results from the german spondyloarthritis inception cohort. Arthritis Rheum. 2009;60(3):717-727. doi:10.1002/art.24483

15. Economou C, Kaitelidou D, Karanikolos M, Maresso A. Greece: Health System Review. Health Syst Transit. 2017;19(5):1166.

16. Ko S, Chi C, Yeh M, Wang S, Tsai Y HM. Lifestyle changes for treating psoriasis. Cochrane Database Syst Rev. 2019;7:CD011972. doi:10.1002/14651858.CD011972.pub2

17.Jois RN, Macgregor AJ, Gaffney K. Recognition of inflammatory back pain and ankylosing spondylitis in primary care. Rheumatology. 2008;47(9):1364-1366. doi:10.1093/rheumatology/ken224

18. National Institute for Health and Care Excellence. Spondyloarthritis overview - NICE Pathways. 2019. https:// pathways.nice.org.uk/pathways/spondyloarthritis. Accessed July 23, 2019.

19. Bhosle MJ, Kulkarni A, Feldman SR, Balkrishnan R. Quality of life in patients with psoriasis. Health Qual Life Outcomes. 2006;4:35. PMID:16756666.

20. Percival J, McGregor C. An Evaluation of Understandability of Patient Journey Models in Mental Health. JMIR Hum factors. 2016;3(2):e20. doi:10.2196/humanfactors.5640

21. Health Equality Europe. Understanding Health Technology Assessment (HTA). 2008. https://htai.org/wp-content/ uploads / 2018/02/PCISG-Resource-HEE_ENGLISH_ PatientGuidetoHTA_Jun14.pdf. Accessed July 23, 2019.

22. Mumtaz A, FitzGerald O. Application of the GRAPPA Psoriatic Arthritis Treatment Recommendations in Clinical Practice. Curr Rheumatol Rep. 2010;12(4):264-271. doi:10.1007/s11926-010-0109-y

23. López-Estebaranz JL, de la Cueva-Dobao P, de la Torre Fraga C, et al. Manejo de la psoriasis moderada-grave en condiciones de práctica habitual en el ámbito hospitalario español. Actas Dermosifiliogr. 2018;109(7):631-642. doi:10.1016/j.ad.2018.02.015

24. Atreja A, Bellam N, Levy SR. Strategies to enhance patient adherence: making it simple. MedGenMed. 2005;7(1):4. PMID:16369309.
PROVENANCE AND PEER REVIEW

Not commissioned; externally peer reviewed. 